REGARDS

SUR L'ECONOMIE ALLEMAND

BULLETIN ECONOMIQUE DU CIRAC
Regards sur l'économie allemande

Bulletin économique du CIRAC

$91 \mid 2009$

Varia

\title{
Une récession historique
}

Isabelle Bourgeois

\section{OpenEdition}

Journals

Édition électronique

URL : http://journals.openedition.org/rea/3668

DOI : 10.4000/rea.3668

ISBN : 978-2-8218-0878-2

ISSN : 1965-0787

Éditeur

CIRAC

Édition imprimée

Date de publication : 1 mai 2009

Pagination : 3-4

ISSN : 1156-8992

Référence électronique

Isabelle Bourgeois, "Une récession historique », Regards sur l'économie allemande [En ligne], 91 | mai 2009, mis en ligne le 20 mai 2009, consulté le 15 septembre 2020. URL : http://

journals.openedition.org/rea/3668 


\title{
Une récession historique
}

La RFA connaît la plus forte récession de son histoire, selon le rapport de printemps des instituts de conjoncture. Plus ouverte aux échanges que les autres économies européennes, et spécialisée dans la production de biens d'investissements comme de biens de consommation durables, l'économie allemande subit de plein fouet la chute accélérée de la demande extérieure. Les instituts, qui ne prévoyaient encore à l'automne qu'une contraction modérée de son PIB, envisagent maintenant un recul de $6 \%$ pour 2009, de même que le gouvernement fédéral. Si, comme la plupart des observateurs, instituts et gouvernement considèrent que le point bas devrait être dépassé au cours de l'année, ils n'attendent pas de stabilisation de la conjoncture allemande avant le milieu de 2010. L'évolution de l'économie mondiale étant peu prévisible, ils restent prudents dans leurs prévisions pour l'an prochain $(-0,5 \%)$.

\begin{abstract}
Une des raisons de cette prudence, qui avait motivé le refus de l'institut DIW de communiquer une prévision chiffrée (et lui a valu d'être vivement critiqué par ses collègues), est le caractère inédit de la récession globale actuelle qui défie tous les modèles mathématiques, ceux-ci ne permettant que d'extrapoler les données récoltées par le passé. Or la globalisation a considérablement changé la donne. Au défaut d'instruments macro-économiques adéquats s'ajoute une pression médiatique accrue se transmuant en ce que les Instituts incriminent dans leur rapport comme une "surenchère des révisions par le bas ». Or, expliquent-ils à l'intention du DIW, même si cela peut sembler scientifiquement justifié, refuser de produire des estimations chiffrées est absurde. D'une part, c'est irréaliste dans une société fondée sur la liberté d'expression et où règne une forte demande d'informations de la part des milieux économiques et politiques. D'autre part, un tel refus de la part d'instances scientifiques indépendantes serait hautement dommageable, puisque non seulement il sèmerait le doute chez les acteurs économiques, mais surtout parce qu'il ouvrirait en grand la voie à la désinformation (qu'elle émane d'entreprises, de partis politiques ou même du gouvernement en cette phase électorale) et jetterait de surcroît le discrédit sur les prévisions du gouvernement en rendant « impossible leur comparaison avec celles d'instituts indépendants ».
\end{abstract}

En Allemagne, la production industrielle s'est contractée bien plus fortement que dans les autres pays industrialisés: depuis février 2008, elle s'est réduite d'un cinquième (Bundesbank). Le ralentissement de l'activité des divers partenaires commerciaux s'est traduit par un recul des commandes de biens intermédiaires, puis de biens d'investissements ; la construction mécanique a ainsi réduit en janvier et février sa production de 18,5\% par rapport à la fin 2008 (Buba). La baisse des exportations (7,5\% au dernier semestre 2008 ; Instituts) a finalement amené les entreprises à réduire leurs investissements (près de $5 \%$ ), mais tardivement, le taux d'utilisation des capacités étant resté à un niveau assez élevé jusqu'à la fin de l'été dernier du fait du stock de commandes accumulées. C'est alors seulement qu'a pris fin la phase d'expansion de l'investissement qui durait depuis 2003, expliquent les Instituts. L'origine de la baisse est bien la crise de la demande, car les Instituts ne constatent pas de restriction généralisée du crédit.

La consommation intérieure s'est révélée en Allemagne " un facteur de stabilisation », à la différence des pays affectés de surcroît par une crise immobilière, soulignent les Instituts. La baisse des prix pétroliers, le recul de l'inflation à partir de l'été dernier ont en effet rehaussé le pouvoir d'achat, de même que la poursuite de la décrue du chômage jusqu'en décembre a entretenu un climat favorable. Bien que la production industrielle ait chuté, le marché de l'emploi ne s'est pas détérioré. II y a surtout deux raisons à cela : les services ne sont guère touchés par le recul de l'activité, et dans l'industrie, le chômage partiel (670 000 salariés en mars ; Bundesagentur) a visiblement rempli jusqu'ici sa fonction d'amortisseur social de la crise (voir REA 89/09). Mais le taux de chômage (7,8 \% en mars et avril en critères OIT selon l'Agence de Nuremberg) devrait augmenter significativement dès la fin 2009 pour s'établir à plus de $10 \%$ en 2010 (Instituts).

Pour l'avenir, les Instituts esquissent deux tendances. Si, comme le pense le FMI, la finance mondiale ne surmonte pas rapidement ses difficultés et provoque une nouvelle crise de confiance, un risque déflationniste n'est pas à exclure. Mais il est tout aussi probable que « la conjoncture allemande redémarrera plus vite que prévu ». Certains indicateurs plaident en ce sens : I'amélioration du bilan de certaines banques, de même que les effets positifs à attendre de la politique économique nationale et mondiale.

La récession actuelle ayant pour seule origine une crise financière, la priorité immédiate pour le gouvernement allemand est de stabiliser le système bancaire, y compris au besoin par des nationalisations, rappellent les Instituts, " car plus vite sa situation sera normalisée, plus vite d'autres mesures de politique économique amélioreront les perspectives conjoncturelles ; notamment en accroissant l'efficacité de la politique monétaire ».
Chute de la production industrielle et de l'investissement

Le marché du travail tient bon pour l'instant

L'économie allemande pourrait redémarrer plus vite que prévu

Priorité absolue à la stabilisation du système bancaire... 
Les deux programmes conjoncturels adoptés (voir REA 90/09) contiennent des mesures de soutien à la relance (hausse des investissements publics, légère baisse des prélèvements) et peuvent de ce fait être en toute légitimité financés par la dette. II n'en va pas de même des mesures « s'assimilant à des subventions ou destinées à donner un coup de pouce à la consommation au prix d'une distorsion de la concurrence ", comme la «prime à la casse » qui accentuera l'an prochain la récession dans l'automobile ou l'aide à des entreprises en difficulté qui pénalise les plus performantes.

\begin{tabular}{|c|c|c|c|c|}
\hline \multicolumn{5}{|c|}{ Prévisions du rapport de printemps 2009 du groupe de travail des Instituts économiques } \\
\hline & 2007 & 2008 & 2009 & 2010 \\
\hline \multicolumn{5}{|c|}{ (variation en \% par rapport à la même période de l'année précédente) } \\
\hline $\begin{array}{l}\text { PIB réel } \\
\text { Ouest (Berlin inclus) } \\
\text { Est } \\
\text { Consommation privée } \\
\text { Consommation publique } \\
\text { Investisssements bruts } \\
\text { biens d'équipement } \\
\text { construction } \\
\text { autres } \\
\text { Demande intérieure } \\
\text { Exportations } \\
\text { Importations } \\
\text { Prix à la consommation }(2000=\text { indice 100) } \\
\text { Coûts salariaux unitaires }\end{array}$ & $\begin{array}{r}2,5 \\
2,5 \\
2,5 \\
-0,4 \\
2,2 \\
4,3 \\
6,9 \\
1,8 \\
8,0 \\
1,1 \\
7,5 \\
5,0 \\
2,3 \\
0,4\end{array}$ & $\begin{array}{r}1,3 \\
1,3 \\
1,1 \\
-0,1 \\
2,0 \\
4,4 \\
5,9 \\
3,0 \\
6,6 \\
1,7 \\
2,7 \\
4,0 \\
2,6 \\
2,1\end{array}$ & $\begin{array}{r}-6,0 \\
\cong-6 \\
\cong-5 \\
0,3 \\
2,3 \\
-8,7 \\
-16,4 \\
-3,9 \\
2,0 \\
-0,8 \\
-22,6 \\
-13,3 \\
0,4 \\
2,8\end{array}$ & $\begin{array}{r}-0,5 \\
\cong-0,5 \\
\cong-1 \\
-1,2 \\
2,0 \\
0,4 \\
-2,1 \\
1,9 \\
2,0 \\
-0,7 \\
2,4 \\
1,9 \\
0,0 \\
-1,3\end{array}$ \\
\hline \multicolumn{5}{|c|}{ (chiffres nominaux) } \\
\hline $\begin{array}{l}\text { Déficit public en \% du PIB } \\
\text { Déficit public (en milliards } € \text { ) } \\
\text { Actifs (en millions) } \\
\text { Chômeurs indemnisés (en millions) }\end{array}$ & $\begin{array}{l}-0,2 \\
-4,2 \\
39,8 \\
3,78\end{array}$ & $\begin{array}{l}-0,1 \\
-3,3 \\
40,3 \\
3,27\end{array}$ & $\begin{array}{r}-3,7 \\
-89,2 \\
39,8 \\
3,72\end{array}$ & $\begin{array}{r}-5,5 \\
-132,5 \\
38,7 \\
4,69\end{array}$ \\
\hline
\end{tabular}

Source des données : Gemeinschaftsdiagnose Frühjahr 2009 (23-04-2009). Les prévisions prennent pour hypothèse notamment un prix moyen du baril de

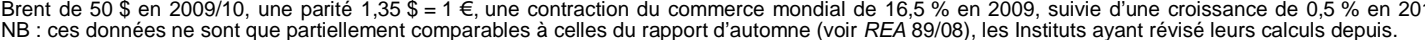

... et à la préservation du rating de l'Allemagne
Et le gouvernement doit résister à la tentation, très forte en année électorale, de lancer un troisième programme conjoncturel ; les effets des deux précédents sur l'état des finances publiques sont impossibles à chiffrer, le volume requis par le soutien au système bancaire n'étant pas connu. Enfin, il est impératif de renouer avec la consolidation budgétaire, entre autres pour préserver le rating de la RFA. Et, dès que possible, cette consolidation devra se faire par une réduction des dépenses ; toute hausse des prélèvements fiscaux et sociaux " réduirait les perspectives de croissance ».

LE MORAL DES ALLEMANDS RESTE ÉTONNAMmENT SOLIDE dans cette récession historique. Les indices ZEW (finance) et ifo (industrie) tendent vers le haut depuis plusieurs mois ; l'indice GfK révèle que les consommateurs gardent confiance. Certes, les experts prévoient près de 5 millions de chômeurs en 2010, mais contrairement à la menace que brandissent à l'approche des élections quelques personnalités de gauche comme Oskar Lafontaine ou Michael Sommer (DGB), aucun vaste mouvement social ne se profile à I'horizon. II est vrai qu'outre-Rhin, les conflits se règlent par négociation entre partenaires sociaux. Et, comme le formule Berthold Huber (IG Metall), « nous cherchons [au contraire] à éviter les mouvements sociaux en nous mettant au travail pour résoudre les problèmes ». Et il décrit ainsi la culture sociale allemande : "les salariés ont un grand intérêt à ce que le développement de leur entreprise soit durable » (Die Welt, 26-04-09). Si quelques grands patrons sont sur la sellette, il n'en va pas de même de l'écrasante majorité de leurs collègues, ces patrons des PME qui sont la base même de l'économie et qui, comme le souligne une étude de l'Institut der Deutschen Wirtschaft, sont "les gardiens de l'économie sociale de marché ». C'est peut-être là qu'il faut chercher la source du courage pragmatique des acteurs économiques allemands : dans leur sens des réalités et leur culture du long terme (voir REA 84/07). Et dans la confiance que leur confère une approche collective pour résoudre les difficultés. "L'Etat seul ne pourra pas y parvenir. Tous doivent apporter leur contribution ", disait la chancelière Merkel lors d'une cérémonie commémorant les 60 ans de la RFA et les 20 ans de la chute du Mur (Handelsblatt, 04-05-09). L'Histoire allemande est une longue suite de chocs et ruptures ; il semblerait qu'elle ait développé chez nos voisins une capacité d'adaptation qui leur permet d'affronter l'adversité en toute conscience et sans perdre confiance.

Isabelle Bourgeois (08-05-2009) 